\title{
LHC, New Physics and Neutrinos
}

\author{
Frank F. Deppisch ${ }^{* \dagger}$ \\ University College London, United Kingdom \\ E-mail: f.deppisch@ucl.ac.uk
}

Models of neutrino mass generation constitute well motivated scenarios of Beyond-the-StandardModel physics. The interplay between high energy collider physics and low energy searches provides us with an effective approach to rule out, constrain and pinpoint such models. In this report, we give a brief overview of examples where collider searches at the LHC can help determine the mechanism of light neutrino mass generation and potentially falsify baryogenesis mechanisms.

Neutrino Oscillation Workshop

4 - 11 September, 2016

Otranto (Lecce, Italy)

\footnotetext{
* Speaker.

$\dagger$ The author would like to thank the organizers of the NOW2016 Workshop for the opportunity to contribute to the conference and the proceedings.
} 


\section{Introduction}

Following the discovery of the Higgs, we are close to verifying the mechanism of charged fermion mass generation. What remains missing is an understanding of the light neutrino masses. The observation of neutrino oscillations shows that neutrinos have finite masses and that lepton flavour is violated. Neutrinos are also popularly considered to be Majorana particles, an assumption that facilitates an understanding of their small masses. It is natural to expect that the violation of the individual lepton flavours and, in the case of Majorana neutrinos, the total lepton number will show up in other contexts as well. This includes rare lepton flavour violating (LFV) decays of muons/taus and the total lepton number violating (LNV) neutrinoless double beta $(0 v \beta \beta)$ decay.

Quite generally, the possible violation of lepton flavour/number should be searched for at all energies that are experimentally accessible. This is because the observation of such processes would equally allow us a direct insight into the mechanism of neutrino mass generation. The most popular example is the so called seesaw mechanism (of type I) in which heavy right-handed Majorana neutrinos $N$ with masses $\gtrsim 10^{11} \mathrm{GeV}$ are added to the Standard Model (SM). Their Yukawa coupling with the left-handed neutrinos induces the light Majorana masses of light neutrinos after electroweak (EW) symmetry breaking. This motivates the lightness of neutrinos through the breaking of lepton number symmetry at a very high scale [1].

Despite its popularity, the generic seesaw mechanism has major phenomenological issues: (i) In the regime $m_{N} \gtrsim 10^{11} \mathrm{GeV}$, heavy neutrinos are far too heavy to be probed experimentally; (ii) Heavy neutrinos are sterile and they only interact through a small mixing with light neutrinos. In this short proceedings report, we will briefly review two scenarios that instead include $\mathrm{TeV}$ scale and potentially non-singlet neutrinos and which can be probed at the LHC. In addition, we will comment on the general impact of the experimental observation of LNV on baryogenesis models.

\section{Low Scale Seesaw}

In the standard type-I seesaw model with the (one generation) mass matrix for the left- and right-handed neutrino, the mass of the light neutrino $v$ and its mixing $\theta$ with the heavy neutrino $N$ is given by $m_{v}=-m_{D}^{2} / m_{N}$ and $\theta=m_{D} / m_{N}=\sqrt{m_{v} / m_{N}}$, respectively. Here, $m_{D}$ is the neutrino Dirac mass, expected to be of the order of the EW scale, and $m_{N}$ is an LNV Majorana mass of the right-handed neutrino. For an observed light neutrino mass scale $m_{v} \approx 0.1 \mathrm{eV}$ this yields $\theta \approx 10^{-5} \sqrt{\mathrm{GeV} / m_{N}}$. For a $\mathrm{GeV}$ to $\mathrm{TeV}$ scale for the heavy neutrino mass, the mixing is rather small. This will be very different in the inverse seesaw scenario [2] described by the mass matrix

$$
\left(\begin{array}{ccc}
0 & m_{D} & 0 \\
m_{D} & \mu_{R} & m_{N} \\
0 & m_{N} & \mu_{S}
\end{array}\right),
$$

similarly for the left-handed neutrino, the right-handed neutrino and an additional SM gauge singlet state $S$. Due to the presence of the small lepton number violating mass parameters $\mu_{R}$ and $\mu_{S}$, light neutrino masses are achievable for any $\theta=m_{D} / m_{N}$ [3]; in the simplest inverse scenario with $\mu_{R}=0$ one has $\theta \approx 10^{-2} \sqrt{\mathrm{keV} / \mu_{S}}$. The reason for this suppression can be understood as the two heavy neutrino states formed by $N$ and $S$ have opposite $C P$ parities and they combine to form quasi-Dirac 

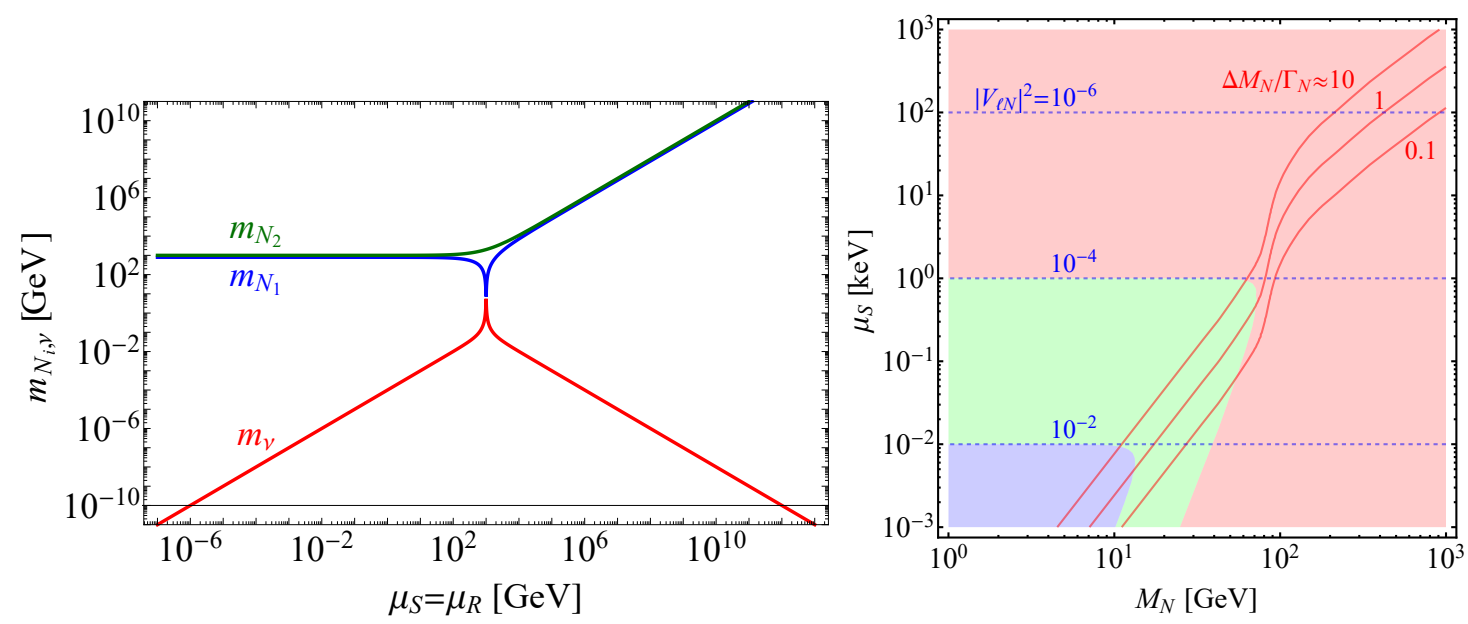

Figure 1: Left: Neutrino mass spectrum as function of $\mu_{S}=\mu_{R}\left(m_{N}=1 \mathrm{TeV}, m_{D}=10 \mathrm{GeV}\right)$. Right: Ratio of heavy neutrino mass splitting to decay width in the inverse seesaw model (solid red contours). The shaded regions take into account the effect of both the mass and coupling suppression with an LNV signal too small to be observed in the red region.

neutrinos with a fractional mass splitting of order $\mu_{S} / m_{N}$. All lepton number violating observables, such as the light neutrino mass, will be suppressed by this small mass splitting.

In order to see the transition between standard and inverse seesaw, we choose $\mu_{S}=\mu_{R}$ in eq. (2.1). Fixing the other terms as $m_{N}=1 \mathrm{TeV}$ and $m_{D}=10 \mathrm{GeV}$, Fig. 1 (left) shows that successful light neutrino mass generation occurs for $\mu \approx 10^{-6} \mathrm{GeV}$ corresponding to the inverse seesaw and $\mu \approx 10^{12} \mathrm{GeV}$ corresponding to the normal high-scale seesaw. The inverse case contains two heavy Majorana neutrinos with masses $m_{N} \pm \mu_{S}$ constituting a quasi-Dirac state, whereas for large $\mu \gg m_{N} \mathrm{GeV}$ two heavy quasi-degenerate Majorana neutrinos with masses $\mu \pm m_{N}$ are formed.

\section{Heavy Sterile Neutrinos}

A large number of laboratory searches put constraints on the mixing between sterile and active neutrinos: For $m_{N} \ll 1 \mathrm{MeV}$, sterile neutrinos are being probed in neutrino oscillation experiments. For pure Majorana sterile neutrinos, $0 v \beta \beta$ searches provide stringent constraints on the mixing with electron neutrinos $[5,6]$, but these limits are considerably weakened for quasi-Dirac neutrinos such as found in the inverse seesaw mechanism discussed above. For $1 \mathrm{MeV} \lesssim m_{N} \lesssim 1 \mathrm{GeV}$, the active-sterile mixing is constrained by peak searches in leptonic decays of pions and kaons and in beam dump experiments. See [4] for a more complete overview of experimental searches.

Regarding LNV at high energy colliders, a general observation can be made in scenarios with approximately conserved lepton number like the above inverse seesaw mechanism: Like any LNV observable, the rate of an LNV process will be suppressed by the small mass splitting, but for onshell resonant production of a heavy neutrino, the suppression is with respect to the neutrino width, $\Delta m_{N} / \Gamma_{N}$, rather than the absolute mass or the energy scale of the process. For $\Delta m_{N} \approx \Gamma_{N}$ it can be resonantly enhanced [7]. The effect of the suppression is shown in Fig. 1 (right) giving contours of $\Delta m_{N} / \Gamma_{N}$ as a function of the inverse seesaw parameters $m_{N}$ and $\mu_{S}$. Within the red shaded region 


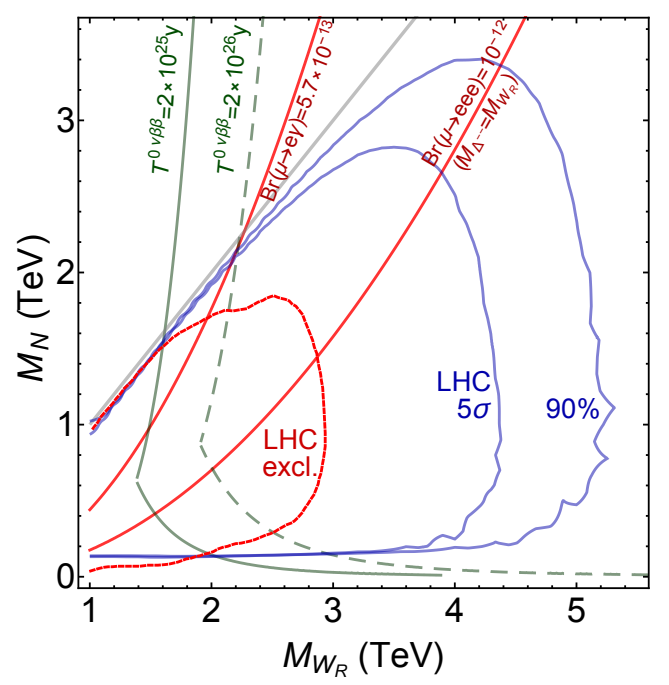

Figure 2: LHC sensitivity of heavy neutrino production via right-handed currents. Solid blue contours give the signal significance at $14 \mathrm{TeV}$ and $\mathscr{L}=300 \mathrm{fb}^{-1}$. Green and red contours show the sensitivity of $0 v \beta \beta$ and LFV searches.

the suppression by either the mass splitting or the active-sterile mixing $\left|V_{l N}\right|^{2}$ would be too severe to expect an LNV observation in the near future.

In the specific context of the LHC, a Majorana heavy neutrino leads to a LNV signature with two same-sign leptons plus jets and no missing energy: $p p \rightarrow W^{(*)} \rightarrow N \ell^{ \pm} \rightarrow \ell^{ \pm} \ell^{ \pm} j j$ [8]. The CMS and ATLAS collaborations have performed direct searches for the production of heavy neutrinos limiting the mixing to active neutrinos $\left|V_{e(\mu) N}\right|^{2} \lesssim 10^{-2}-10^{-1}$ for $m_{N} \lesssim 500 \mathrm{GeV}$ at $\sqrt{s}=8 \mathrm{TeV}$ [9]. During the ongoing run II of the LHC, the limits could be improved to apply to about a TeV. In addition to the basic $s$-channel production, it is also very worthwhile to consider other production modes and decay scenarios: Electroweak $t$-channel processes of the form $p p \rightarrow W^{*} \gamma^{*} \rightarrow N \ell^{ \pm} j j$ can for example give a better sensitivity for higher $m_{N}$ values.

\section{Left-Right Symmetric Models}

One of the simplest options to extend the above sterile neutrino scenario is an additional, broken $U(1)^{\prime}$ gauge symmetry under which the heavy neutrinos are charged. Under favourable parameter conditions, heavy neutrinos can then be pair-produced abundantly and be probed even for very small mixing with the active neutrinos [10]. Another popular option are left-right symmetric models (LRSMs); the minimal LRSM extends the SM gauge symmetry to $S U(2)_{L} \times S U(2)_{R} \times$ $U(1)_{B-L}$ [11]. Leptons are assigned to doublets $L=(v, \ell)_{L}$ and $R=(N, \ell)_{R}$ under $S U(2)_{L}$ and $S U(2)_{R}$, respectively. The Higgs sector of the minimal LRSM consists of a bidoublet and two triplets $\Delta_{L, R}$. The VEV $v_{R}$ of the neutral component of $\Delta_{R}$ breaks the gauge symmetry $S U(2)_{R} \times$ $U(1)_{B-L}$ to $U(1)_{Y}$ and gives masses to the RH gauge bosons $W_{R}, Z_{R}$ and the right-handed neutrinos $N$. LRSMs provide a simple ultraviolet complete seesaw mechanism with the key properties built in: The presence of right-handed neutrinos is a necessary ingredient and the LNV seesaw scale can be identified with the breaking scale of the $S U(2)_{R}$ symmetry. 

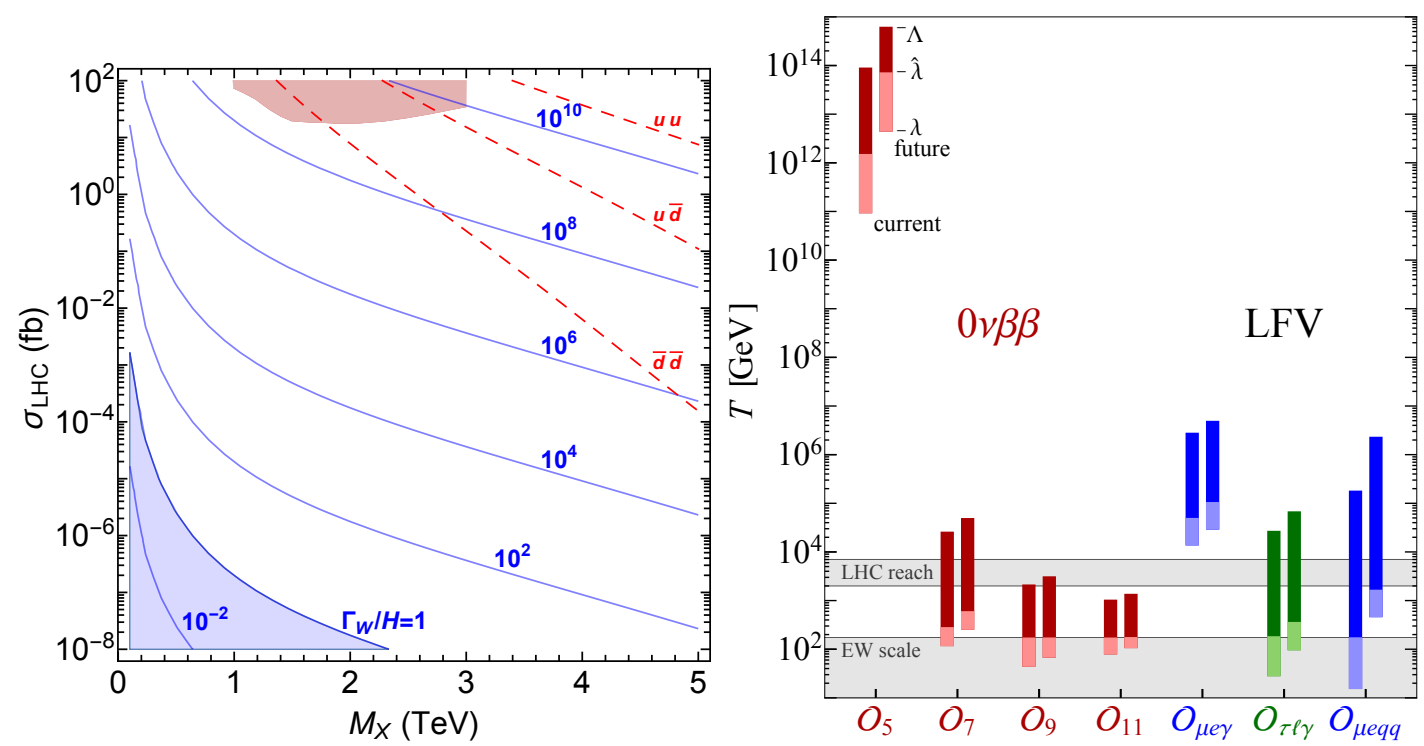

Figure 3: Left: Lepton number washout rate as function of the corresponding LHC cross section and resonance mass (solid blue contours). The dashed red curves give typical cross sections of resonances produced through the quark channels $u u, u \bar{d}$ and $\bar{d} \bar{d}$ with gauge coupling strength for comparison. Taken from [16]. Right: Temperature intervals where the given LNV and LFV operators are in equilibrium assuming that the corresponding process is observed at the current or future experimental sensitivity. Taken from [17].

With regard to LHC searches, the right-handed current interactions in the LRSM can lead to a significant enhancement of the LNV signal. Even for negligible left-right neutrino mixing, heavy neutrinos can be directly produced via $s$-channel $W_{R}$ exchange $[12,13]$. Fig. 2 (left) compares the sensitivity of such searches with the sensitivity of $0 v \beta \beta$ and low energy LFV experiments assuming equality of the $S U(2)_{L}$ and $S U(2)_{R}$ gauge couplings, $g_{R}=g_{L}$.

\section{Falsifying High-Scale Baryogenesis}

The observed matter-antimatter asymmetry of the universe cannot be understood with Standard Model physics. A large number of possible mechanisms to generate the observed asymmetry have been proposed. An interesting scenario is leptogenesis [14]. In its original formulation, the out-of-equilibrium and $C P$ violating decay of the heavy Majorana neutrinos in the type-I seesaw mechanism create a lepton asymmetry which is then converted into a baryon asymmetry through $(B+L)$ violating electroweak sphaleron processes [15].

The presence of lepton number violation (LNV) is a crucial ingredient in leptogenesis. Vice versa, the observation of LNV would have important consequences on the viability of baryogenesis models in general; specifically, it is possible to falsify a large class of high-scale baryogenesis scenarios if LNV was observed at the LHC [16]. For example if a resonant LNV process with the signature $p p \rightarrow l^{ \pm} l^{ \pm} j j$ is observed, its LHC cross section $\sigma_{\mathrm{LHC}}$ is related to the induced lepton asymmetry washout rate $\Gamma_{W} / H$ (relative to the expansion of the universe) [16],

$$
\log _{10}\left(\Gamma_{W} / H\right) \gtrsim 6.9+0.6\left(M_{X} / \mathrm{TeV}-1\right)+\log _{10}\left(\sigma_{\mathrm{LHC}} / \mathrm{fb}\right) .
$$




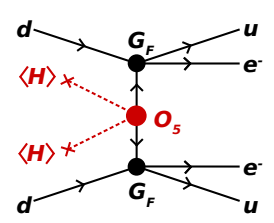

(a)

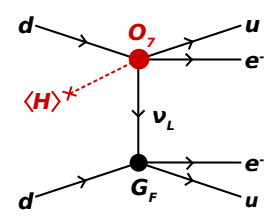

(b)

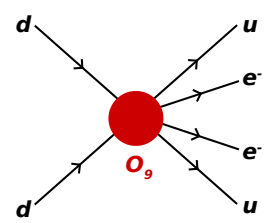

(c)

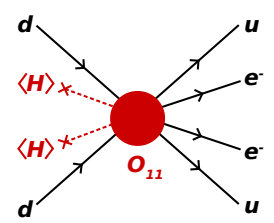

(d)

Figure 4: Contributions to $0 v \beta \beta$ decay generated by effective dimension-5 (a), dimension-7 (b), dimension9 (c) and dimension-11 (d) operators.

Here $M_{X}$ is the mass of the hypothetically observed resonance. If $\Gamma_{W} / H \gg 1$, the dilution of a primordial net lepton number density, understood to be produced in a baryogenesis mechanism at a higher scale, is highly effective and the lepton asymmetry would be washed out before sphaleron processes take effect. This is also illustrated in Fig. 3 (left) showing $\Gamma_{W} / H$ as a function of the observed LNV resonance mass $M_{X}$ and the process cross section $\sigma_{L H C}$. Observation of LNV at the LHC would therefore strongly constrain baryogenesis scenarios above the scale $M_{X}$.

A similar argument can be applied to non-standard mechanisms mediating $0 v \beta \beta$ decay and low energy LFV processes [17]: if observed, the corresponding processes would be in equilibrium in certain temperature ranges. The low energy $0 v \beta \beta$ decay can in general be triggered by effect LNV operators of dimension 5 (the Weinberg operator leading to the standard light neutrino exchange), dimension 7 (leading to long-range contributions again mediated by light neutrinos) and higher odd-dimensional operators (leading to short range operators). Typical contributions, up to dimension 11, are graphically displayed in Fig. 4. Current $0 v \beta \beta$ decay searches probing half lives $T_{1 / 2}^{0 v \beta \beta} \gtrsim 10^{26} \mathrm{yr}$ are roughly sensitive to the scales $\Lambda_{5} \approx 10^{14} \mathrm{GeV}$ (seesaw scale), $\Lambda_{7} \approx 10^{4} \mathrm{GeV}$ and $\Lambda_{9,11} \approx 10^{3} \mathrm{GeV}$, of these operators. Similarly, the presence of any of these operators will also lead to a washout of lepton number in the early universe. This is shown in Fig. 3 (right) where the coloured bars denote the efficient equilibration temperatures assuming the relevant observable is seen at the current (left bar) or expected future $\left(T_{1 / 2}^{0 v \beta \beta} \approx 10^{27} \mathrm{yr}\right.$, right bar) sensitivity. In the case of the 7,9,11-dimensional effective operators $\mathscr{O}_{7,9,11}$ mediating $0 v \beta \beta$ decay, an electron lepton asymmetry present at energies higher than the electroweak scale would be washed out.

Observation of LFV via 6-dimensional lepton flavor violating (LFV) operators at compatible scales would allow to extend the argument to other flavours than the electron. The most stringent limits on LFV are currently set on 6-dimensional $\Delta L=0$ operators $\mathscr{O}_{\mu e \gamma}, \mathscr{O}_{\tau l \gamma}$ and $\mathscr{O}_{\mu e q q}$ leading to the following decays and current experimental limits: $\mathrm{Br}_{\mu \rightarrow e \gamma}<5.7 \times 10^{-13}, \mathrm{Br}_{\tau \rightarrow \ell \gamma} \lesssim 4.0 \times 10^{-8}$ $(\ell=e, \mu), \mathrm{R}_{\mu \rightarrow e}^{\mathrm{Au}}<7.0 \times 10^{-13}$. The expected sensitivities of ongoing and planned experiments are $\mathrm{Br}_{\mu \rightarrow e \gamma} \approx 6.0 \times 10^{-14}, \mathrm{Br}_{\tau \rightarrow \ell \gamma} \approx 1.0 \times 10^{-9}$ and $\mathrm{R}_{\mu \rightarrow e}^{\mathrm{Al}} \approx 2.7 \times 10^{-17}$. In a similar analysis to the above discussed LNV operators, one can relate the rare LFV process rate with the corresponding flavor equilibration temperature range. In Fig. 3 (right), the flavor equilibration ranges are shown assuming current and future LFV process sensitivities, alongside the electron lepton asymmetry washout of the $0 v \beta \beta \mathrm{LNV}$ operators. If LNV and LFV is present at similar scales, the washout of the electron lepton number will thus be transmitted to muons or taus.

Searches for LNV in $0 v \beta \beta$ decay, at the LHC and in other contexts (for example LNV meson decays) are thus powerful tools to narrowing down models of baryogenesis. If $0 v \beta \beta$ decay was observed via a non-standard mechanism, it would point us to low-scale baryogenesis as well as a 
probable discovery of LNV at the LHC. If however, high-scale baryogenesis is realised in nature, no LNV is expected to be discovered at the LHC. If $0 v \beta \beta$ decay was observed, its underlying mechanism is then likely to be the standard mass mechanism via the Weinberg operator and it would point us to a high-scale origin of neutrino masses. Loop holes in this reasoning exist and we would like to refer the reader to $[16,17]$ for a more detailed discussion.

\section{Conclusions}

The synergy between low energy/high intensity searches and high energy LHC searches are an effective approach to rule out, constrain and ideally pinpoint neutrino mass models. In this report we have briefly reviewed a few phenomenological scenarios where the LHC can help to determine whether the light neutrino masses, and maybe the matter-antimatter asymmetry as well, are generated in a mechanism close to the electroweak scale or beyond.

\section{References}

[1] P. Minkowski, Phys.Lett. B67, 421 (1977); M. Gell-Mann, P. Ramond, and R. Slansky, (1979), Print-80-0576 (CERN); R. N. Mohapatra and G. Senjanović, Phys. Rev. Lett. 44, 912 (1980); J. Schechter and J. W. F. Valle, Phys. Rev. D22, 2227 (1980); J. Schechter and J. W. F. Valle, Phys. Rev. D25, 774 (1982).

[2] R. N. Mohapatra and J. W. F. Valle, Phys. Rev. D34, 1642 (1986).

[3] M. C. Gonzalez-Garcia and J. W. F. Valle, Phys. Lett. B216, 360 (1989).

[4] F. F. Deppisch, P. S. Bhupal Dev, and A. Pilaftsis, New J. Phys. 17, 075019 (2015), 1502.06541.

[5] S. Kovalenko, Z. Lu, and I. Schmidt, Phys.Rev. D80, 073014 (2009), 0907.2533.

[6] A. Atre, T. Han, S. Pascoli, and B. Zhang, JHEP 0905, 030 (2009), 0901.3589.

[7] A. Pilaftsis, Nucl.Phys. B504, 61 (1997), hep-ph/9702393.

[8] A. Pilaftsis, Z.Phys. C55, 275 (1992), hep-ph/9901206.

[9] CMS Collaboration, V. Khachatryan et al., 1501.05566.

[10] F. F. Deppisch, N. Desai, and J. W. F. Valle, Phys.Rev. D89, 051302 (2014), 1308.6789.

[11] J. C. Pati and A. Salam, Phys. Rev. D10, 275 (1974); R. N. Mohapatra and J. C. Pati, Phys.Rev. D11, 2558 (1975); G. Senjanovic and R. N. Mohapatra, Phys.Rev. D12, 1502 (1975).

[12] W.-Y. Keung and G. Senjanovic, Phys. Rev. Lett. 50, 1427 (1983).

[13] M. Nemevsek, F. Nesti, G. Senjanovic, and Y. Zhang, Phys.Rev. D83, 115014 (2011), 1103.1627; J. Aguilar-Saavedra, F. Deppisch, O. Kittel, and J. Valle, Phys.Rev. D85, 091301 (2012), 1203.5998;

S. Das, F. Deppisch, O. Kittel, and J. Valle, Phys.Rev. D86, 055006 (2012), 1206.0256.

[14] M. Fukugita and T. Yanagida, Phys. Lett. B174, 45 (1986).

[15] V. A. Kuzmin, V. A. Rubakov, and M. E. Shaposhnikov, Phys. Lett. B155, 36 (1985).

[16] F. F. Deppisch, J. Harz, and M. Hirsch, Phys.Rev.Lett. 112, 221601 (2014), 1312.4447; F. F. Deppisch and J. Harz, (2014), 1408.5351.

[17] F. F. Deppisch, J. Harz, M. Hirsch, W.-C. Huang, and H. Päs, Phys. Rev. D92, 036005 (2015), 1503.04825 . 